\title{
BMJ Open Cost-effectiveness of bedaquiline, pretomanid and linezolid for treatment of extensively drug-resistant tuberculosis in South Africa, Georgia and the Philippines
}

\author{
Gabriela Beatriz Gomez (D) ,1,2 Mariana Siapka, ${ }^{1,3}$ Francesca Conradie, ${ }^{4}$ \\ Norbert Ndjeka (1) , ${ }^{5}$ Anna Marie Celina Garfin, ${ }^{6}$ Nino Lomtadze, ${ }^{7}$ Zaza Avaliani, ${ }^{7}$ \\ Nana Kiria, ${ }^{7}$ Shelly Malhotra, ${ }^{8,9}$ Sarah Cook-Scalise, ${ }^{8,10}$ Sandeep Juneja, ${ }^{8}$ \\ Daniel Everitt, ${ }^{8}$ Melvin Spigelman, ${ }^{8}$ Anna Vassall ${ }^{1}$
}

To cite: Gomez GB, Siapka M, Conradie F, et al. Costeffectiveness of bedaquiline, pretomanid and linezolid for treatment of extensively drug-resistant tuberculosis in South Africa, Georgia and the Philippines. BMJ Open 2021;11:e051521. doi:10.1136/ bmjopen-2021-051521

- Prepublication history and additional supplemental material for this paper are available online. To view these files, please visit the journal online (http://dx.doi.org/10.1136/ bmjopen-2021-051521).

Received 18 April 2021 Accepted 08 November 2021

Check for updates

(C) Author(s) (or their employer(s)) 2021. Re-use permitted under CC BY. Published by BMJ.

For numbered affiliations see end of article.

Correspondence to Dr Gabriela Beatriz Gomez; gabriela.gomez@Ishtm.ac.uk

\section{ABSTRACT}

Objectives Patients with highly resistant tuberculosis have few treatment options. Bedaquiline, pretomanid and linezolid regimen (BPaL) is a new regimen shown to have favourable outcomes after six months. We present an economic evaluation of introducing $B P a L$ against the extensively drug-resistant tuberculosis (XDR-TB) standard of care in three epidemiological settings.

Design Cost-effectiveness analysis using Markov cohort model.

Setting South Africa, Georgia and the Philippines.

Participants XDR-TB and multidrug-resistant tuberculosis (MDR-TB) failure and treatment intolerant patients.

Interventions BPaL regimen.

Primary and secondary outcome measures (1) Incremental cost per disability-adjusted life years averted by using BPaL against standard of care at the Global Drug Facility list price. (2) The potential maximum price at which the $\mathrm{BPaL}$ regimen could become cost neutral.

Results BPaL for XDR-TB is likely to be cost saving in all study settings when pretomanid is priced at the Global Drug Facility list price. The magnitude of these savings depends on the prevalence of XDR-TB in the country and can amount, over 5 years, to approximately US\$ 3 million in South Africa, US\$200 000 and US\$ 60000 in Georgia and the Philippines, respectively. In South Africa, related future costs of antiretroviral treatment (ART) due to survival of more patients following treatment with $\mathrm{BPaL}$ reduced the magnitude of expected savings to approximately US\$ 1 million. Overall, when BPaL is introduced to a wider population, including MDR-TB treatment failure and treatment intolerant, we observe increased savings and clinical benefits. The potential threshold price at which the probability of the introduction of $\mathrm{BPaL}$ becoming cost neutral begins to increase is higher in Georgia and the Philippines (US\$ 3650 and US\$ 3800 , respectively) compared with South Africa (US\$ 500) including ART costs.

Conclusions Our results estimate that $\mathrm{BPaL}$ can be a cost-saving addition to the local TB programmes in varied programmatic settings.

\section{Strengths and limitations of this study}

- We are presenting consistent cost-saving results that are conservative. We are likely to have underestimated secondary benefits, particularly in terms of transmission averted.

- Our results are based on recently collected cost data that are setting specific, therefore are highly relevant for local policy-makers.

- Key limitations include a restriction to a health service perspective and our results being based on efficacy estimates from a small study without a randomised control group.

\section{INTRODUCTION}

Patients with extensively drug-resistant tuberculosis (XDR-TB) and those who have failed or are intolerant to their multidrug-resistant tuberculosis (MDR-TB) treatment have few treatment options, low cure rates and high mortality. ${ }^{1}$ Treatment and management of such patients is costly to the health system and patients (with high hospitalisation rates for long periods and high drug costs). ${ }^{2}$ Available treatments are also difficult for patients to use due to the complex and significant side effects and adverse events as well as the number of drugs prescribed, often including a combination of injectables and oral medications, depending on the setting. ${ }^{3}$ Use of new drug pretomanid in the bedaquiline, pretomanid and linezolid (BPaL) regimen was approved by the US Food and Drug Administration (FDA) under the Limited Population pathway for Antibacterial and antifungal Drugs pathway in 2019 and conditionally approved by the European Medical Agency (EMA) and Drugs Controller General of 
India, among othersDemocratic Republic (DR) of the Congo, Georgia, South Africa, Tajikistan, Turkmenistan, Uzbekistan and Zimbabwe to date) ${ }^{4-6}$ It was also prequalified by WHO. The BPaL regimen was also endorsed for use under operational research conditions by the WHO. ${ }^{7}$ This regimen consists of three drugs, is shorter in duration compared with standard therapy and is administered orally. ${ }^{8}$ The BPaL regimen was shown to lead to favourable outcomes after 6 months in a high percentage of patients with highly drug-resistant forms of tuberculosis in an open-label, single-group study at three South African sites in the Nix-TB trial. ${ }^{9}$ There were associated side effects observed, primarily due to the high dose of linezolid studied. The long-term follow-up of this study recently showed sustained efficacy. ${ }^{10}$ An alternative dosing scheme was explored in the phase III ZeNix trial. ${ }^{11}$ The results of ZeNix were presented in July 2021 at the International AIDS Conference. They demonstrated that BPaL remains effective in patients with XDR-TB and those who have failed or are intolerant to their MDR-TB treatment with either reduced dosage or duration of the linezolid component of the regimen in sites across Georgia, Moldova, Russia and South Africa. With the maintenance of efficacy, there was a decrease in linezolid-associated side effects. ${ }^{12}$

Following these empirical results, countries may consider the health benefits and economic trade-offs of including this regimen in national recommendations. To date, a series of economic evaluations looking at the addition of bedaquiline to MDR treatment regimens for the treatment of adult patients with pulmonary MDR-TB and/or XDR-TB have been published. ${ }^{13-22}$ However, there has been no economic evaluation of a shorter, alloral treatment regimen, consisting of three drugs with minimal pre-existing resistance, such as BPaL. We present here an economic evaluation of introducing BPaL for use in XDR-TB against the local standard of care. We consider the eligible population with and without MDR-TB failure or intolerant patients in three epidemiological settings.

\section{METHODS}

We developed a Markov cohort model to estimate cost and benefits of BPaL for treatment of a cohort of diagnosed patients with XDR-TB (with and without the inclusion of MDR-TB failure and intolerant patients) in three settings adopting a lifetime horizon and a health sector perspective. We parameterised this model with a combination of publicly available and aggregated cost and health outcome data by setting. Because we used only secondary data sources without any identifiable information and publicly available, this study was exempt from submission to ethics committee. We chose a Markov cohort model, as opposed to a decision tree, to be able to model both disease and treatment processes where timing of events and repeat events are important. In such a modelling framework, the impact of transmission on the wider population has been excluded, which may underestimate health impact. The model schematic is provided in online supplemental figure S1.

Our main analytical outcomes are (1) the incremental costs and disability-adjusted life years (DALYs) averted of using BPaL against the context-specific standard of care at the Global Drug Facility list price and (2) the potential maximum drug price at which BPaL could be considered cost saving in three epidemiological settings.

\section{Population and setting}

The primary population modelled is patients with XDRTB. We also explored in a separate scenario the inclusion of those patients who have failed or are intolerant to their MDR-TB treatments, as this is part of the approved label by the US FDA and EMA. XDR-TB is defined as resistance to any fluoroquinolone and to at least one of three second-line injectable drugs (capreomycin, kanamycin and amikacin), in addition to MDR-TB, defined as resistance to two first-line TB drugs, isoniazid and rifampicin. MDR-TB treatment failure is defined as nonresponsiveness to MDR-TB treatment at 6 months (lack of sputum conversion by the end of the intensive phase) and intolerance is defined as the inability to continue a second-line drug regimen due to a documented intolerance to para-aminosalicylic acid, ethionamide, aminoglycosides or fluoroquinolones. We chose to separate these two scenarios because those patients who have failed an MDR-TB regimen containing bedaquiline may not be considered eligible for $\mathrm{BPaL}$ without further drug sensitivity testing ${ }^{23-25}$ and considering that the definition of XDR-TB has been recently updated as to include MDR-TB that is resistant to a fluoroquinolone and at least one of bedaquiline or linezolid (or both). We performed the analysis for three countries chosen to represent a range of epidemiological settings across two dimensions: prevalence of MDR-TB and HIV coinfection (epidemiological profiles introduced in online supplemental table S1). These are drivers of observed differences in both burden and mortality from XDR-TB. In South Africa, we observe a high burden of both MDR/XDR-TB and HIV; in Georgia, there is high burden of MDR/XDR-TB but a lower HIV burden; while in the Philippines, there is a low incidence of XDR-TB and HIV. ${ }^{3}$

\section{Intervention, comparator and outcomes}

The intervention considered is a shortened (6 months), all-oral regimen for treatment of patients with XDR$\mathrm{TB}$, including $\mathrm{BPaL}$, see figure 1 . This duration can be extended to 9 months in the case of no sputum conversion after 4 months of treatment. ${ }^{9}$ We modelled the published prescription recommendations for pretomanid as well as current guidance for monitoring of bedaquiline and linezolid in the definition of tests and visits schedules. ${ }^{26-29}$

We characterised the comparator using standardised recommendations for XDR-TB regimens (18 months) that may be modified based on drug sensitivity testing results. Routine clinical practice as defined in current national guidelines and validated with local expert 
Standard of care for Georgia and The Philippines
Standard of care for South Africa
Intervention care: $\mathrm{BPaL}$

6 months: LZD - BDQ - DLM - CFZ - hdINH (or ETO)
12 months: LZD - CFZ - hdINH (or ETO)
Monthly visits, PHC doctor
ECG at initiation, week 2, then monthly during BDQ
Repeat ECG 3-monthly if CFZ and no BDQ
FBC and neutrophil count at initiation, week 2, then
monthly during LZD
Audiometry at baseline and month 3

6 months: LZD - BDQ - DLM - CFZ - TRD - Z-
hdINH (or ETO)
12 months: LZD - CFZ - TRD - Z - hdINH (or ETO)
Monthly visits, PHC doctor
ECG at initiation, week 2, then monthly during BDQ
Repeat ECG 3-monthly if CFZ and no BDQ
FBC and neutrophil count at initiation, week 2, then
monthly during LZD
Sputum liquid culture monthly if TRD
Audiometry at baseline and month 3

6 months: LZD - BDQ - DLM - CFZ - TRD - Z hdINH (or ETO)

Monthly visits, $\mathrm{PHC}$ doctor Repeat ECG 3-monthly if CFZ and no BDQ
initiation, week 2, then monthly during BDQ monthly during LZD

Audiometry at baseline and month 3

Figure 1 Characteristics of the standard of care and intervention modelled by setting. BDQ, bedaquiline; BPaL, bedaquiline, pretomanid and linezolid; CFZ, clofazimine; DLM, delamanid; ETO, ethionamide; FBC, full blood count; hdINH, high-dose Isoniazid; LZD, linezolid; PHC, primary healthcare; PZA, pyrazinamide; TRD, terizidone.

opinion in each setting is the comparator. Comparators by setting are detailed in figure 1 .

Partial lung resection for patients with XDR-TB is not included for either the intervention or comparator as, after assessing a recent review of the evidence, it was not recommended. ${ }^{30}$

We used information from the Nix-TB study, an open-label single-arm study, to inform clinical efficacy of the intervention (ClinicalTrials.gov reference: NCT02333799). ${ }^{9}$ The Nix-TB study aimed to evaluate the efficacy, safety, tolerability and pharmacokinetics of BPaL after six to 6-9 months of treatment in XDR-TB or treatment intolerant or non-responsive MDR-TB. It had a total of 109 patients enrolled. After 6-month follow-up from the end of treatment, it showed that $90 \% \quad(95 \%$ CI, 83 to 95 ) of participants had a favourable outcome. Side effects included peripheral neuropathy in $81 \%$ of patients and myelosuppression in $48 \%$. All these were manageable and led often to dose reductions or interruptions of linezolid treatment. The occurrence of these side effects led to further investigation on how to optimise the linezolid dose. In the ZeNix trial (ClinicalTrials. gov reference: NCT03086486), ${ }^{12}$ the efficacy observed in Nix-TB was confirmed with less side effects, from $84 \%$ to 93\% depending on the linezolid dosage across the four arms of the trial.

For state transitions in the Markov cohort model beyond the trial time period and the effectiveness of the comparator, we use national secondary data. Expert elicitation was used where no data were available, for example, estimating the extent to which patients return to care after default. Cure in the standard of care has been defined as reported treatment completed, as recommended by national policies. A patient is classified as having completed treatment if there is no evidence of failure and three or more consecutive negative cultures taken at least 30 days apart after the intensive phase. In the Nix-TB trial, cure was defined as no evidence of infection 6 months after end of treatment. ${ }^{9}$ Failure is a lack of conversion by the end of the intensive phase or bacteriological reversion in the continuation phase after conversion to negative at the end of the intensive phase. Patients can die at any time and while being in any state. The number of DALYs averted was the measure of quality and length of life chosen to assess health outcomes. DALYs averted are suitable for comparisons across economic evaluations in lowincome and middle-income countries consistent with the recommendations of the International Decision Support Initiative Reference Case for Economic Evaluation. ${ }^{31}$ DALYs averted were calculated as the sum of the present value of future years of life lost through TB mortality and the present value of years adjusted for disability caused by TB using the standard formula. ${ }^{32}$ Disability weights were sourced from the Global Burden of Disease study in $2017^{33}$ and are presented in table 1 .

\section{Costs}

We used cost estimates from the literature and local consultations and guidelines to build disaggregated unit costs for the intervention and comparator (ie, drugs, visits, tests). We did not collect new data but obtained data from the Global Health Costing Consortium database and from VALUE TB (a multicountry TB costing study funded by the Bill and Melinda Gates Foundation) ${ }^{34}{ }^{35}$ Unrelated costs and the costs of comorbidities have been excluded, except for costs related to HIV in South Africa, which have a significant impact on the intervention in this setting and are presented separately. All costs are presented in 2018 US\$, after conversion from other currencies per standard recommendations. ${ }^{36}$ Parameters and assumptions made are presented in tables 1 and 2.

We modelled treatment outcomes for 5 years but capture all costs and consequences relevant to the economic evaluation until death. We followed international conventions and discounted both cost and effects at $3 \%$ for our main analysis. ${ }^{31}$

\section{Uncertainty and sensitivity analysis}

Uncertainty has been explored using deterministic and probabilistic sensitivity analyses. A deterministic sensitivity analysis is presented to evaluate the main drivers of the results, both in terms of cost savings and DALYs averted. We conducted a sensitivity analysis around discounting assumptions in addition to other parameters. 
Table 1 Input parameters for cost-effectiveness analyses

\begin{tabular}{|c|c|c|c|c|}
\hline & South Africa & Georgia & The Philippines & Reference \\
\hline \multicolumn{5}{|l|}{ Population } \\
\hline MDR intolerant/failure & \multicolumn{3}{|c|}{$10 \%$ of all patients with MDR-TB } & Assumption \\
\hline HIV prevalence $(n=56 / 109)^{\star}$ & $51 \%$ & - & - & 9 \\
\hline Age (years) & \multicolumn{3}{|c|}{35 (range 17-60) } & 9 \\
\hline \multicolumn{5}{|l|}{ Treatment outcomes $\dagger$} \\
\hline Per cent completed at $18 \mathrm{mo}$, SoC & $0.73(0.031)$ & 0.585 & $0.64-0.73$ & $45-48$ \\
\hline Per cent failure at $18 \mathrm{mo}, \mathrm{SoC}$ & $0.045(0.015)$ & 0.073 & $0.045(0.015)$ & 454748 \\
\hline Per cent LTFU at $18 \mathrm{mo}$, SoC & $0.10(0.021)$ & 0.219 & $0.15-0.20$ & 454748 \\
\hline Per cent completed/cure at $6 \mathrm{mo}, \mathrm{BPaL}$ & \multicolumn{3}{|c|}{$0.90(0.83-0.95)$} & 9 \\
\hline Per cent death, BPaL $(n=7 / 109)$ & \multicolumn{3}{|l|}{$0.064(0.026)$} & 9 \\
\hline Per cent LTFU at 6 mo, BPaL & \multicolumn{3}{|l|}{$0.04(0.021)$} & 47 \\
\hline \multicolumn{5}{|l|}{ Outcomes after treatment } \\
\hline Risk of relapse $\neq$ & \multicolumn{3}{|c|}{$2836(2131-3693)$} & 49 \\
\hline $\begin{array}{l}\text { Per cent return to care after relapse, SoC and } \\
\text { BPaL }\end{array}$ & \multicolumn{3}{|l|}{$0.75( \pm 20 \%)$} & Assumption \\
\hline $\begin{array}{l}\text { Per cent return to care after LTFU, SoC and } \\
\text { BPaL }\end{array}$ & \multicolumn{3}{|l|}{$0.20( \pm 20 \%)$} & Assumption \\
\hline $\begin{array}{l}\text { Median survival after treatment failure, mo (LTFU/ } \\
\text { relapse/palliative), SoC and BPaL }\end{array}$ & \multicolumn{3}{|c|}{$19.84(4.16-26.04)$} & 50 \\
\hline \multicolumn{5}{|l|}{ Disability weight } \\
\hline XDR-TB/MDR-TB, without HIV infection & \multicolumn{3}{|c|}{$0.333(0.224-0.454)$} & 33 \\
\hline XDR-TB/MDR-TB, with HIV infection & \multicolumn{3}{|c|}{$0.408(0.274-0.549)$} & 33 \\
\hline HIV/AIDS receiving ART without TB & \multicolumn{3}{|c|}{$0.078(0.052-0.111)$} & 33 \\
\hline
\end{tabular}

*All HIV-positive patients are assumed to be on/started on ART.

†Transformed to a rate (per mo) assumed constant.

łIncidence risk of relapse per 100000 successfully treated.

ART, antiretroviral treatment; BPaL, bedaquiline, pretomanid and linezolid; LFTU, loss to follow-up; MDR-TB, multidrug-resistant tuberculosis; mo, months; n, number; SoC, standard of care; TB, tuberculosis; XDR-TB, extensively drug-resistant tuberculosis.

The probabilistic sensitivity analysis was done by running the model 10000 times while sampling from the parameter's distributions. We present these analyses for two outcomes. First, for the incremental cost per DALY averted of using BPaL against standard of care at the Global Drug Facility list price, we plotted the cost-effectiveness planes by country, illustrating the uncertainty in both costs estimates and DALYs averted. Second, for the potential maximum price at which the BPaL regimen could still be considered cost saving, we present threshold price estimates as curves, plotting the probability of the regimen being cost saving as a function of drug price.

\section{Patient and public involvement}

There was no direct involvement of patients or the public in this health economics modelling study.

Table 2 Input cost estimates for cost-effectiveness analyses (US\$2018 per month)

\begin{tabular}{|c|c|c|c|c|}
\hline & South Africa & Georgia & The Philippines & Reference \\
\hline BPaL & $\begin{array}{l}296.4 \text { (drugs) } \\
65.3 \text { (delivery) }\end{array}$ & $\begin{array}{l}214.0 \text { (drugs) } \\
31.0 \text { (delivery) }\end{array}$ & $\begin{array}{l}214.0 \text { (drugs) } \\
38.3 \text { (delivery) }\end{array}$ & $3551-55$ \\
\hline Antiretroviral treatment & 249.2 & - & - & 57 \\
\hline
\end{tabular}

*Average of $10 \%$ hospice inpatient unit; $40 \%$ community care and $50 \%$ no care.

$\mathrm{BPaL}$, bedaquiline, pretomanid and linezolid. 


\section{RESULTS}

\section{Costs, effectiveness and cost-effectiveness}

When assessing the potential cost and effectiveness of introducing BPaL for the treatment of XDR-TB against the current standard of care, we observe that in all three settings this regimen has the potential to be cost saving at the current Global Drug Facility list price (ie, US\$ 364 per treatment course for pretomanid) ${ }^{37}$ Results are presented in table 3. These savings are a function of the cost of care and the magnitude of XDR-TB burden. Savings following the introduction of $\mathrm{BPaL}$ are more important in settings with more expensive current standard of care or a higher burden of XDR-TB. Consequently, cost savings are estimated greater in South Africa and Georgia than in the Philippines.

The magnitude of the potential savings increased when the clinical indication for BPaL was modelled to include those patients who are MDR-TB treatment intolerant or MDR-TB treatment failures. In a setting such as South Africa, where changes in treatment guidelines for TB may have significant cost consequences across both TB and HIV programmes, we have included both TB costs and costs due to continuous antiretroviral treatment (ART) following principles of good practice in economic evaluation. ${ }^{31}$ As a result, the savings in this high HIV prevalence setting are reduced once we consider the increase in lifetime ART costs (following more patients surviving the TB episode and needing ART for more years of life). Even with this conservative assumption, $\mathrm{BPaL}$ introduction in a setting such as South Africa continues to be estimated as cost saving. These additional (HIV-related) costs are not significant in low HIV settings such as Georgia or the Philippines.

In addition to the main results in terms of incremental costs and DALYs averted, we also present several sensitivity analyses. All deterministic sensitivity analyses can be found inonline supplemental tables S2-S4 and online supplemental figures S2-S8. In general, a programme's performance in terms of rates of loss to follow-up or mortality during treatment will drive the value for money of introducing BPaL. For example, in South Africa, we observe that our costs results are most sensitive to variations in assumptions regarding the performance of the TB programme. BPaL introduction ceases to be cost saving in scenarios where we assume a long term under performance of the standard of care (such as higher mortality rates at 18 months or a higher rate of loss to follow-up at 18 months) compared with the base case assumptions. In Georgia and the Philippines, our cost results are robust to the assumptions tested with $\mathrm{BPaL}$ introduction remaining cost saving across sensitivity analyses. Effectiveness results are sensitive to changes in assumptions for mortality and loss to follow-up for both $\mathrm{BPaL}$ and standard of care across settings. A threshold analysis explores the probability of introducing $\mathrm{BPaL}$ becoming cost neutral (ie, not cost saving) as a function of possible prices (figure 2). This is based on the results from the probabilistic sensitivity analyses, which can be found in the online supplemental file. We observed that the potential threshold price at which the probability of the introduction of BPaL becoming cost neutral begins to increase is higher in Georgia and the Philippines (US\$ 3650 and US $\$ 3800$, respectively) compared with South Africa (US\$ 500).

\section{DISCUSSION}

BPaL for treatment of XDR-TB is likely to be cost saving in the study settings at the proposed price. In settings such as South Africa, related future costs like those from the HIV programme (ART costs) may reduce the magnitude of expected savings to the health service. $\mathrm{BPaL}$ treatment is estimated to avert more deaths in patients with XDR-TB compared to the current standard of care. The reduction of expected savings relates to these patients requiring lifelong ART.

Cost savings from the introduction of the $\mathrm{BPaL}$ regimen are higher in settings with a more expensive current standard of care. Consequently, the threshold price at which $\mathrm{BPaL}$ becomes cost neutral is higher in less expensive settings: US\$ 3650 and US\$ 3800 for Georgia and the Philippines, respectively, and US $\$ 500$ for South Africa for our base case of only patients with XDR-TB, after factoring in incremental cost of ART. It is worth noting that this threshold price is not the price at which the introduction of such regimen will still be considered cost-effective, it indicates an increasing probability of a price being cost neutral.

The impact of BPaL on costs and DALYs averted depends on the programmatic performance of the standard of care. A standard of care with lower loss to follow-up or mortality rates than currently assumed will decrease the value for money of introducing BPaL. Overall, when BPaL is introduced to a larger patient population (including MDR-TB treatment failure and treatment intolerant), we observe an increase in the incremental benefits, that is, an increase in deaths averted and in DALYs averted. The increase in benefits observed is due to both the shortening of the regimen (principal driver of cost savings) and the better curative performance in clinical trials compared with the current standard of care.

In this assessment, we established a conservative baseline. We are likely to have underestimated secondary benefits, particularly in terms of transmission averted. We assessed standard of care scenarios that are less expensive (with more community/outpatient treatment) than previously observed, which is an emerging treatment norm. ${ }^{38}$ A standard of care including more hospitalisation time in all these settings would result in higher savings following the introduction of BPaL. In addition, our analysis was restricted to a health service perspective. Costs incurred by patients and their households have been shown to be a significant burden to society. ${ }^{39}$ In limiting the perspective to the health service, we aimed to be conservative in the analysis of cost savings and its relationship to price. 


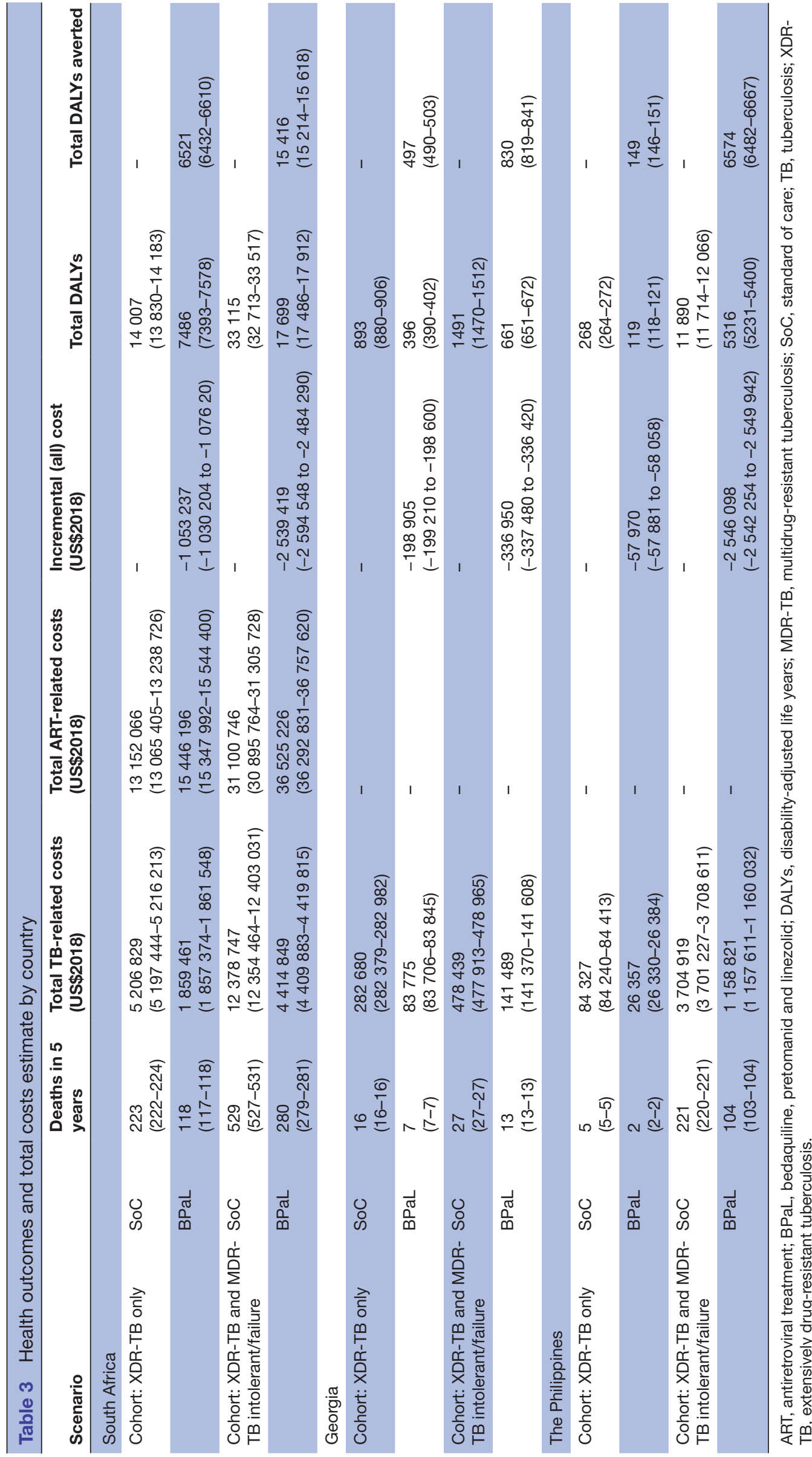



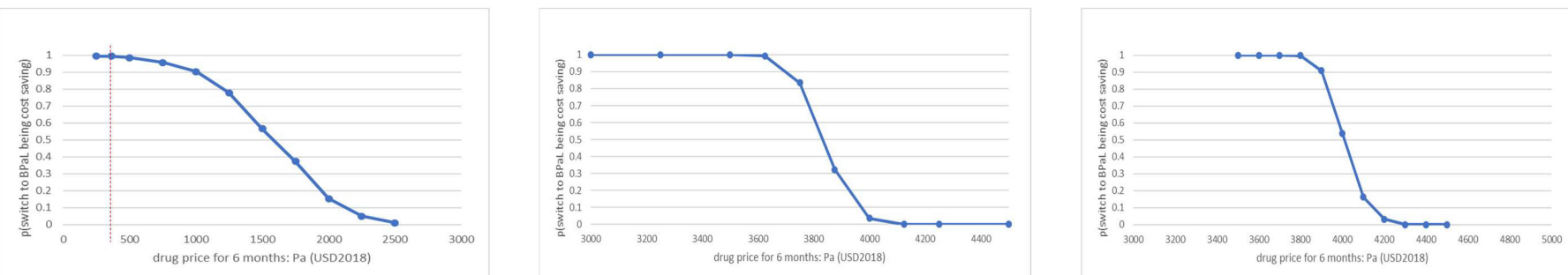

Figure 2 Probability of BPaL being cost saving as the price estimates for pretomanid change. Dashed line corresponds to US\$ 364 per treatment course for pretomanid. BPaL, bedaquiline, pretomanid and linezolid; p, probability; Pa, pretomanid.

However, in doing so, we may have underestimated the value of this regimen to society. Finally, we did not consider costs and/or effects of introducing this new technology in the countries. The introduction of a new technology implies fixed costs of training, changes in guidance and changes in systems, among others. This omission may have led to an underestimation of the BPaL costs.

This study has important limitations. First, this study was based on efficacy estimates from a small study $(n=109)$ without a randomised control group. During this clinical study, both bedaquiline and linezolid were increasingly used as part of the standard of care to treat both MDR-TB and XDR-TB (all the patients received bedaquiline and $81 \%$ also received linezolid), as reflected in our comparison arm. Previous evidence has shown that while bedaquiline had a low incidence of adverse events leading to permanent drug discontinuation, linezolid had a high incidence. ${ }^{40}$ In the Nix-TB study, a high percentage of patients had adverse events related to linezolid during the study. However, all eight patients who had the regimen interrupted for adverse events resumed and completed the full 26 weeks of treatment. ${ }^{9}$ Due to both the small sample size and the use of linezolid in both comparator groups, we were not able to quantify the impact on DALYs or costs of adverse events. However, the efficacy results of the Nix-TB study have been recently confirmed in a phase III trial, the ZeNix study which included other settings. ${ }^{12}$ This linezolid dose optimisation trial also demonstrated that the linezolid dose and/or duration of the regimen will likely reduce, which will potentially make our results an underestimate of savings. Finally, cost parameter values were estimated from guidelines and verified against empirically measured recent estimates from a multinational and standardised costing study, improving the comparability of the results across settings. This comparison showed that our estimates were lower than those empirically measured. The implication being that the cost savings presented here could be considered as conservative.

\section{CONCLUSIONS}

The optimisation of MDR/XDR-TB regimens is a priority in global health. Our study is the first model to explore the costs and benefits of introducing an all-oral shorter treatment regimen for XDR-TB treatment using data from recent trials. Other economic evaluations alongside clinical trials are expected to be published soon looking at other shorter combinations, including the PRACTECAL trial which has published positive initial data. ${ }^{41-43}$

In November 2019, the $\mathrm{WHO}^{44}$ reviewed clinical data available for Nix-TB following the recommendation from the FDA and concluded that more research was needed before the programmatic implementation of the regimen worldwide could be recommended. Currently, WHO guidelines endorse the use of the $\mathrm{BPaL}$ regimen under operational research conditions in MDR-TB patients with $\mathrm{TB}$ that is resistant to fluoroquinolones. At the time of writing, operations research had commenced in six countries and expected to start in another five/six countries by late 2021 . This operational research will be useful for countries to further assess costs and benefits of introducing BPaL. Until then, our results point to BPaL being an efficient and needed addition to the local TB programmes in varied programmatic settings.

\section{Author affiliations}

${ }^{1}$ Department of Global Health and Development, Faculty of Public Health and Policy, London School of Hygiene and Tropical Medicine, London, UK

${ }^{2}$ Modelling, Epidemiology and Data Science Department, Sanofi Pasteur, Lyon, France

${ }^{3}$ Impact Epilysis, Thessaloniki, Greece

${ }^{4}$ Clinical HIV Research Unit, University of the Witwatersrand, Johannesburg, South Africa

${ }^{5}$ National TB Programme, South Africa Department of Health, Pretoria, Gauteng, South Africa

${ }^{6}$ National Tuberculosis Control Program, Bureau of Disease Prevention and Control, Department of Health, Manila, The Philippines

${ }^{7}$ Department of TB Surveillance and Strategic Planning, National Center for

Tuberculosis and Lung Diseases, Tbilisi, Georgia

${ }^{8}$ TB Alliance, New York, New York, USA

${ }^{9} \mathrm{Global}$ Access, International AIDS Vaccine Initiative (IAVI), New York, New York, USA

${ }^{10}$ TB Division, USAID, Washington, DC, USA

Correction notice This article has been corrected since it was published Online First. The funding statement has been updated.

Twitter Norbert Ndjeka @NNdjeka

Acknowledgements The authors are grateful to all patients, healthcare professionals and national tuberculosis programme staff who contributed time and effort to make the initial studies possible.

Contributors GBG contributed to the study design, model development, analysis and manuscript drafting and editing. MaS contributed to the study design, model development and manuscript editing. FC, NN, AMCG, NL, ZA and NK contributed to 
the model parameterisation, analysis and manuscript editing. SM, SC-S, SJ, DE and MeS contributed to the analysis and manuscript editing. AV contributed to the study design, model development, analysis and manuscript editing. All authors have read and approved the final version of the manuscript.GBG is the guarantor of the paper.

Funding This work was supported by the TB Alliance (grant number LSHTM/GATB 16003) and in whole or in part, by the Bill \& Melinda Gates Foundation.

Competing interests GG is currently employed by Sanofi Pasteur as decision science expert. Sanofi Pasteur has not provided funding for this work. SM was employed by the TB Alliance at the start of this project. SC-S, SJ, DE and MS are employees of the TB Alliance.

Patient consent for publication Not applicable.

Provenance and peer review Not commissioned; externally peer reviewed.

Data availability statement All data relevant to the study are included in the article or uploaded as supplementary information. The data used in the paper are based on the sources described in the main text and supplementary material and on summarised and published available data from the Nix-TB trial.

Supplemental material This content has been supplied by the author(s). It has not been vetted by BMJ Publishing Group Limited (BMJ) and may not have been peer-reviewed. Any opinions or recommendations discussed are solely those of the author(s) and are not endorsed by BMJ. BMJ disclaims all liability and responsibility arising from any reliance placed on the content. Where the content includes any translated material, BMJ does not warrant the accuracy and reliability of the translations (including but not limited to local regulations, clinical guidelines, terminology, drug names and drug dosages), and is not responsible for any error and/or omissions arising from translation and adaptation or otherwise.

Open access This is an open access article distributed in accordance with the Creative Commons Attribution 4.0 Unported (CC BY 4.0) license, which permits others to copy, redistribute, remix, transform and build upon this work for any purpose, provided the original work is properly cited, a link to the licence is given, and indication of whether changes were made. See: https://creativecommons.org/ licenses/by/4.0/.

\section{ORCID iDs}

Gabriela Beatriz Gomez http://orcid.org/0000-0002-7409-798X

Norbert Ndjeka http://orcid.org/0000-0001-6200-1592

\section{REFERENCES}

1 Collaborative Group for the Meta-Analysis of Individual Patient Data in MDR-TB treatment-2017, Ahmad N, Ahuja SD, et al. Treatment correlates of successful outcomes in pulmonary multidrug-resistant tuberculosis: an individual patient data meta-analysis. Lancet 2018;392:821-34.

2 Global Health Costing Consortium. Unit Cost Study Repository [Internet]. Available: https://ghcosting.org/pages/data/ucsr/app/ [Accessed 05 Jun 2020].

3 World Health Organization. Global tuberculosis report 2019. Geneva: World Health Organization, 2019.

4 Maddipatla M. U.S. FDA approves TB Alliance's treatment for drugresistant tuberculosis. Reuters [Internet], 2019. Available: https:// www.reuters.com/article/us-tb-alliance-fda/u-s-fda-approves-tballiances-treatment-for-drug-resistant-tuberculosis-idUSKCN1V41UU [Accessed 05 Jun 2020].

5 European Medicines Agency. Pretomanid FGK [Internet]. Available: https://www.ema.europa.eu/en/medicines/human/EPAR/pretomanidfgk [Accessed 18 Jan 2021].

6 Reuters. Mylan gets Indian regulatory approval for tuberculosis drug [Internet]. Available: https://www.reuters.com/article/us-mylantuberculosis-india/mylan-gets-indian-regulatory-approval-fortuberculosis-drug-idUSKCN24N19K [Accessed 18 Jan 2021]

7 World Health Organization. WHO consolidated guidelines on tuberculosis. Module 4: treatment - drug-resistant tuberculosis treatment [Internet]. Geneva, 2020. Available: https://apps.who.int/ iris/rest/bitstreams/1280998/retrieve

8 TB Alliance. Our pipeline: BPaL [Internet]. Available: https://www. tballiance.org/portfolio/regimen/bpal [Accessed 05 Jun 2020].

9 Conradie F, Diacon AH, Ngubane N, et al. Treatment of highly drugresistant pulmonary tuberculosis. N Engl J Med 2020;382:893-902.

10 Conradie F, Diacon AH, Ngubane N. Final results of teh NIX-TB clinical study of BPaL regimen for highly resistant TB. virtual, 2021. Available: https://www.croiconference.org/abstract/final-results-ofthe-nix-tb-clinical-study-of-bpal-regimen-for-highly-resistant-tb/
11 TB Alliance. Our pipeline: ZeNix trial [Internet]. Available: https:// www.tballiance.org/portfolio/trial/11883 [Accessed 18 Jan 2021]

12 Conradie F. High rate of successful outcomes treating highly resistant $T B$ in the ZeNix study of pretomanid, bedaquiline and alternative doses and durations of linezolid. Berlin, Germany, 2021.

13 Wolfson LJ, Gibbert J, Wirth D, et al. Cost-Effectiveness of incorporating bedaquiline into a treatment regimen for MDR/XDR-TB in Germany. Eur Respir J 2015;46:1826-9.

14 Wolfson LJ, Walker A, Hettle R, et al. Cost-Effectiveness of adding bedaquiline to drug regimens for the treatment of multidrug-resistant tuberculosis in the UK. PLoS One 2015;10:e0120763.

15 Wolfson L, Wirth D, Jibbert J, et al. Costs and effectiveness of combination therapy with bedaquiline and other anti-tuberculosis drugs in patients with multi- and extensively drug-resistant tuberculosis in Germany. Value Health 2014;17:A674-5.

16 Wolfson L, Walker A, Hettle R, et al. Cost effectiveness of bedaquiline for the treatment of multidrug-resistant tuberculosis. Value Health 2014;17:A595

17 Lu X, Smare C, Kambili C, et al. Health outcomes of bedaquiline in the treatment of multidrug-resistant tuberculosis in selected high burden countries. BMC Health Serv Res 2017;17:87.

18 Schnippel K, Firnhaber C, Conradie F, et al. Incremental cost effectiveness of bedaquiline for the treatment of rifampicin-resistant tuberculosis in South Africa: model-based analysis. Appl Health Econ Health Policy 2018;16:43-54.

19 Schnippel K, Firnhaber C, Page-Shipp L, et al. Impact of adverse drug reactions on the incremental cost-effectiveness of bedaquiline for drug-resistant tuberculosis. Int J Tuberc Lung Dis 2018;22:918-25.

20 Ionescu A-M, Mpobela Agnarson A, Kambili C, et al. Bedaquilineversus injectable-containing drug-resistant tuberculosis regimens: a cost-effectiveness analysis. Expert Rev Pharmacoecon Outcomes Res 2018:18:677-89.

21 Fan Q, Ming W-K, Yip W-Y, et al. Cost-Effectiveness of bedaquiline or delamanid plus background regimen for multidrug-resistant tuberculosis in a high-income intermediate burden city of China. Int $J$ Infect Dis 2019;78:44-9.

22 Mpobela Agnarson A, Williams A, Kambili C, et al. The costeffectiveness of a bedaquiline-containing short-course regimen for the treatment of multidrug-resistant tuberculosis in South Africa. Expert Rev Anti Infect Ther 2020;18:475-83.

23 Kiria N, Avaliani Z, Bolokadze N. Experience of treatment Pre-XDR and XDR-TB/HIV co-infection with bedaquiline and delamanid containing regimens in Georgia. Eur Respir J 2018;52:PA3673.

24 Kiria N, Avaliani Z, Mikiashvili L. Steps of bedaquline implementation in Georgia. Eur Respir J 2016;48:OA3516.

25 Lofranco V. Introduction of bedaquiline in the Philippines. Manila: Crowne Plaza Hotel, 2017

26 Philippine Coalition Against Tuberculosis (PhilCAT), Philippine Society for Microbiology and Infectious Diseases (PSMID), Philippine College of Chest Physicians (PCCP). Task force: clinical practice guidelines for the diagnosis, treatment, prevention and control of tuberculosis in adult Filipinos. Manila, 2016.

27 Republic of South Africa, Department of Health. Interim clinical guidance for the implementation of injectable-free regimens for rifampicin-resistant tuberculosis in adutls, adolescents and children [Internet], 2018. Available: http://www.tbonline.info/media/uploads/ documents/dr_tb_clinical_guidelines_for_rsa_september_2018.pdf

28 Georgia Country Coordinating Mechanism. National Strategy for Tuberculosis Control in Georgia 2019-2022 [Internet]. Tbilisi, 2019. Available: http://www.georgia-ccm.ge/wp-content/uploads/NationalStrategy-for-Tuberculosis-Control-in-Georgia-2019-2022.pdf

29 TB Alliance. Highights of prescribing information [Internet]. New York 2019. Available: https://www.tballiance.org/sites/default/files/assets/ Pretomanid_Full\%20Prescribing\%20Information.pdf

30 World Health Organization. WHO consolidated guidelines on drugresistant tuberculosis treatment. Geneva, 2019.

31 Wilkinson T, Sculpher MJ, Claxton K, et al. The International decision support initiative reference case for economic evaluation: an aid to thought. Value Health 2016;19:921-8.

32 Fox-Rushby JA, Hanson K. Calculating and presenting disability adjusted life years (DALYs) in cost-effectiveness analysis. Health Policy Plan 2001;16:326-31.

33 Global Burden of Disease Collaborative Network. Global burden of disease study 2017 (GBD 2017) disability weights. Seattle, United States: Institute for Health Metrics and Evaluation (IHME), 2018.

34 Global Health Costing Consortium. Unit Cost Study Repository (UCSR) [Internet], 2018. Available: https://ghcosting.org/pages/data/ ucsr/app/ [Accessed 13 Jun 2020].

35 Sweeney S, Cunnama L, Laurence YV. Value TB Dataset: costs per intervention [Internet]. Harv. Dataverse 2021. 
36 Walker D, Kumaranayake L. Allowing for differential timing in cost analyses: discounting and annualization. Health Policy Plan 2002;17:112-8.

37 Stop TB Partnership. The Stop TB Partnership's Global Drug Facility catalog now lists the first-ever, all-oral regimen for highly resistant forms of drug-resistant tuberculosis at US \$1,040 per treatment course [Internet], 2019. Available: http://www.stoptb.org/news/ stories/2019/ns19 025.html [Accessed 18 Jan 2021].

38 Masuku SD, Berhanu R, Van Rensburg C, et al. Managing multidrugresistant tuberculosis in South Africa: a budget impact analysis. int $j$ tuberc lung dis 2020;24:376-82.

39 Laurence YV, Griffiths UK, Vassall A. Costs to health services and the patient of treating tuberculosis: a systematic literature review. Pharmacoeconomics 2015;33:939-55.

40 Lan Z, Ahmad N, Baghaei P, et al. Drug-associated adverse events in the treatment of multidrug-resistant tuberculosis: an individual patient data meta-analysis. Lancet Respir Med 2020;8:383-94.

41 Sweeney S, Gomez G, Kitson N, et al. Cost-effectiveness of new MDR-TB regimens: study protocol for the TB-PRACTECAL economic evaluation substudy. BMJ Open 2020;10:e036599.

42 Rosu L, Madan J, Worrall E. Economic evaluation protocol of a short, all-oral bedaquiline-containing regimen for the treatment of rifampicin-resistant tuberculosis from the stream trial. BMJ Open 2020;10:e042390.

43 Medecins Sans Frontieress. Drug-resistant TB clinical trial ends enrolment early after positive initial data [Internet]. MSF Press Release. Available: https://www.msf.org/drug-resistant-tuberculosistrial-ends-enrolment-after-positive-initial-data [Accessed 20 Aug 2021]

44 World Health Organization. Rapid communication: key changes to the treatment of drug-resistant tuberculosis. Geneva, 2019.

45 Hewison C, Bastard M, Khachatryan N, et al. Is 6 months of bedaquiline enough? results from the compassionate use of bedaquiline in Armenia and Georgia. int $j$ tuberc lung dis 2018;22:766-72.
46 Li Y, Sun F, Zhang W. Bedaquiline and delamanid in the treatment of multidrug-resistant tuberculosis: promising but challenging. Drug Dev Res 2019;80:98-105.

47 Ndjeka N, Schnippel K, Master I, et al. High treatment success rate for multidrug-resistant and extensively drug-resistant tuberculosis using a bedaquiline-containing treatment regimen. Eur Respir $J$ 2018;52:1801528.

48 Department of Health. REVIEW REPORT: 2019 Philippines TB Joint Program Review [Internet], 2019. Available: http://www.ntp.doh.gov. ph/downloads/publications/assessment reports/JPR 2019.pdf

49 Marx FM, Dunbar R, Enarson DA, et al. The temporal dynamics of relapse and reinfection tuberculosis after successful treatment: a retrospective cohort study. Clin Infect Dis 2014;58:1676-83.

50 Pietersen E, Ignatius E, Streicher EM, et al. Long-Term outcomes of patients with extensively drug-resistant tuberculosis in South Africa: a cohort study. Lancet 2014;383:1230-9.

51 Sinanovic E, Ramma L, Vassall A, et al. Impact of reduced hospitalisation on the cost of treatment for drug-resistant tuberculosis in South Africa. Int J Tuberc Lung Dis 2015;19:172-8.

52 Republic of South Africa, Department of Health. Annexure A1 Upfs2018 Tariffs 01 April 2018 Full Paying [Internet], 2018. Available: http://www.health.gov.za/index.php/shortcodes/2015-03-29-10-4247/2015-04-30-09-10-23/uniform-patient-fee-schedule/category/ 448-upfs-2018

53 Republic of South Africa, Department of Health. Master Procurement Catalogue [Internet], 2018. Available: http://www.health.gov.za/index php/component/phocadownload/category/196

54 South African National Blood Service. State Patients Pricelist [Internet]., 2019. Available: https://sanbs.org.za/product-price-list/

55 Stop TB Partnership. GDF Product catalogue [Internet], 2018. Available: http://www.stoptb.org/gdf/drugsupply/catalogue.asp

56 Drenth C, Sithole Z, Pudule E, et al. Palliative care in South Africa. J Pain Symptom Manage 2018;55:S170-7.

57 Meyer-Rath G, van Rensburg C, Chiu C, et al. The per-patient costs of HIV services in South Africa: systematic review and application in the South African HIV investment case. PLoS One 2019;14:e0210497. 